\title{
DISJOINTNESS PRESERVING MAPS BETWEEN VECTOR-VALUED GROUP ALGEBRAS
}

\author{
MALIHEH HOSSEINI AND JUAN J. FONT
}

\begin{abstract}
Let $G$ be a locally compact abelian group and $B$ be a commutative Banach algebra. Let $L^{1}(G, B)$ be the Banach algebra of $B$-valued Bochner integrable functions on $G$. In this paper we provide a complete description of continuous disjointness preserving maps on $L^{1}(G, B)$-algebras based on a scarcely used tool: the vector-valued Fourier transform. We also present necessary and sufficient conditions for these operators to be compact.
\end{abstract}

\section{Introduction}

Linear maps between Banach algebras, Banach lattices, or Banach spaces preserving certain properties have been of a considerable interest for many years. The most classical question concerns isometries, although more recently, maps that preserve spectrum, spectral radius, commutativity, normal elements, self-adjoint elements, nilpotents, idempotents, linear rank, disjointness of cozeroes, or other properties have been intensely investigated.

Among them, maps that preserve the disjointness of cozeroes defined between spaces of scalarvalued continuous functions on locally compact and compact spaces, as a generalization of the concept of homomorphism, have a long history in functional analysis in the context of rings, algebras, or vector lattices under several names such as Lamperti operators, separating maps, disjointness preserving operators, etc. (see, for example, $[1,2,3,4,5,7,8,13,16]$ ). In recent years, certain attention has been given to such maps when defined on spaces of vector-valued continuous functions (see, e.g., $[10,14])$. However, we do not know much about disjointness preserving maps on vectorvalued settings in comparison with scalar-valued contexts and something similar can be said with regard to (algebra) homomorphisms between vector-valued group algebras.

In this paper we focus on the study of disjointness preserving maps defined between vector-valued group algebras. Banach algebras of vector-valued functions date back to the early moments of the theory of Banach algebras and play a natural role in functional analysis. Among them, spaces of

Key words and phrases: Locally compact abelian group, vector-valued group algebras, disjointness preserving mappings.

2010 Mathematics Subject Classification. Primary 47B38; Secondary 43A20, 43A22, 43A25.

J.J. Font is supported by Spanish Government (MTM2016-77143-P), Universitat Jaume I (Projecte P11B2014-35) and Generalitat Valenciana (Projecte AICO/2016/030). 
vector-valued continuous functions and vector-valued group algebras are perhaps the most studied ones. Homomorphisms of algebras ([11]) and multipliers ([22]) on group algebras of vector-valued functions are examples of disjointness preserving maps. Here we provide a weighted composition representation of continuous disjointness preserving maps on vector-valued group algebras and a characterization of compact disjointness preserving maps on the same context. Let us recall that the study of continuous disjointness preserving maps on vector-valued function spaces and the compactness dates back to [15], where Jamison and Rajagopalan described continuous disjointness preserving maps on the Banach space $C(X, E)$ of all continuous functions from a compact space $X$ into a Banach space $E$, and gave criteria of compactness for these maps. Shortly after, in [6], the results of [15] were extended to $C_{0}(X, E)$ for locally compact $X$. It is worth mentioning that our results are based on the vector-valued Fourier transform, a scarcely used tool in the literature, which acts as a "vector-valued Gelfand transform". This technique contrasts with the one used in most previous papers dealing with algebras of vector-valued functions which are based on the scalar-valued Gelfand transform (see e.g., [14] and the references therein).

\section{Preliminaries}

Let $G$ be a locally compact abelian group with the Haar measure $m$ and $B$ be a commutative Banach algebra.

Let $L^{1}(G, B)$ be the Bochner algebra of $G$, i.e., the commutative Banach algebra of integrable functions from $G$ to $B$ endowed with the convolution product

$$
(f * g)(t)=\int_{G} f(t-s) \cdot g(s) d m(s)
$$

for all $f, g \in L^{1}(G, B), t \in G$, and the norm

$$
\|f\|_{1}=\int_{G}\|f(t)\| d m(t)
$$

for all $f \in L^{1}(G, B)$. We shall write $L^{1}(G)$ if $B$ is chosen as the complex numbers. Next we provide the main properties of $L^{1}(G, B)$ and $L^{1}(G)$, which can be found, basically, in [17, Section 4.13] and [20, Chapter 2].

Let $\hat{G}$ be the dual group of $G$. Given $f \in L^{1}(G, B)$, its vector-valued Fourier transform is defined as

$$
\hat{f}(\gamma):=\int_{G} f(t) \cdot \gamma(-t) d m(t)
$$

for a given $\gamma \in \hat{G}$.

Let $A(\hat{G}, B)$ be the vector-valued Fourier algebra associated to $L^{1}(G, B)$, that is,

$$
A(\hat{G}, B):=\left\{\hat{f}: f \in L^{1}(G, B)\right\}
$$


Let us recall that $C_{0}(\hat{G}, B)$ is the Banach space of all continuous $B$-valued functions on $\hat{G}$ vanishing at infinity. It is known that $A(\hat{G}, B) \subset C_{0}(\hat{G}, B)$ separates the points of $\hat{G}$, and that $\|\hat{f}\|_{\infty} \leq\|f\|_{1}$, that is, the Fourier transform, considered as a map from $L^{1}(G, B)$ into $C_{0}(\hat{G}, B)$, is a continuous linear injection. Besides,

(1) $f=0$ if and only if $\hat{f} \equiv 0$ (Uniqueness Principle).

(2) If $f \in L^{1}(G, B)$ (or $\left.L^{1}(G)\right)$ and $g \in L^{1}(G, B)$, then $f * g \in L^{1}(G, B)$ and $\widehat{f * g}=\hat{f} \hat{g}$.

Let $f, g \in L^{1}(G)$ and $b_{1}, b_{2} \in B$. Let us recall that the tensor product of $f$ and $b_{1}$ is defined as $\left(f \otimes b_{1}\right)(\gamma):=f(\gamma) b_{1}$ for all $\gamma \in G$. Here we present some properties of the tensor product:

(1) $f \otimes b_{1} \in L^{1}(G, B)$ and $\left\|f \otimes b_{1}\right\|_{1}=\|f\|_{1}\left\|b_{1}\right\|$.

(2) $\widehat{f \otimes b_{1}}(\gamma)=\left(\hat{f} b_{1}\right)(\gamma)=\hat{f}(\gamma) b_{1}$.

(3) $\left(f \otimes b_{1}\right) *\left(g \otimes b_{2}\right)=(f * g) \otimes b_{1} b_{2}$.

A classical result of Grothendieck asserts that $L^{1}(G, B)$ is isometrically isomorphic to the projective tensor product $L^{1}(G) \hat{\otimes} B$ of $L^{1}(G)$ and $B$. Namely, we can identify

$$
L^{1}(G, B)=\left\{\sum_{i=1}^{\infty} f_{i} \otimes b_{i}: f_{i} \in L^{1}(G), b_{i} \in B, \sum_{i=1}^{\infty}\left\|f_{i}\right\|_{1}\left\|b_{i}\right\|<\infty\right\} .
$$

\section{Disjointness PRESERving MAPS ON VeCtor-VAlued Group ALGeBras}

For $i=1,2$, let $G_{i}$ be locally compact abelian groups and $B_{i}$ be commutative Banach algebras.

Definition 3.1. Let $T: L^{1}\left(G_{1}, B_{1}\right) \longrightarrow L^{1}\left(G_{2}, B_{2}\right)$ be a linear mapping. It is said that $T$ is zero product preserving if $T f * T g=0$ whenever $f * g=0$ for every $f, g \in L^{1}\left(G_{1}, B_{1}\right)$.

Associated to a zero product preserving mapping $T$ we can define a mapping $\hat{T}: A\left(\hat{G}_{1}, B_{1}\right) \longrightarrow$ $A\left(\hat{G}_{2}, B_{2}\right)$ defined as $\hat{T} \hat{f}:=\widehat{T f}$ for all $f \in L^{1}\left(G_{1}, B_{1}\right)$. It is apparent, due to the Uniqueness Principle, that $\hat{T}$ is zero product preserving if and only if so is $T$.

Definition 3.2. Let $T: L^{1}\left(G_{1}, B_{1}\right) \longrightarrow L^{1}\left(G_{2}, B_{2}\right)$ be a linear mapping. It is said that $T$ is separating or disjointness preserving if $\operatorname{coz}(\hat{f}) \cap \operatorname{co} z(\hat{g})=\emptyset$ yields $\operatorname{coz}(\hat{T} \hat{f}) \cap \operatorname{coz}(\hat{T} \hat{g})=\emptyset$ for every $f, g \in L^{1}\left(G_{1}, B_{1}\right)$. If $T$ is a separating bijection whose inverse is also separating, then it is said to be biseparating.

Unless otherwise specified, in the sequel $T: L^{1}\left(G_{1}, B_{1}\right) \longrightarrow L^{1}\left(G_{2}, B_{2}\right)$ will stand for a disjointness preserving map.

It is apparent that, unlike the scalar case, in this vector valued setting, preserving zero products and preserving disjointness of cozeros are different concepts. However if we, for instance, assume that $B_{1}$ and $B_{2}$ are integral domains (i.e., they have no divisors of zero), then both concepts agree. 
Definition 3.3. Let $\hat{G}_{20}:=\left\{\xi \in \hat{G}_{2}: \hat{T} \hat{f}(\xi) \neq 0\right.$ for some $\left.f \in L^{1}\left(G_{1}, B_{1}\right)\right\}$. A point $\gamma \in \hat{G}_{1} \cup\{\infty\}$ is said to be a support point for $\xi \in \hat{G}_{20}$ if for any neighborhood $U$ of $\gamma$, there is $f \in L^{1}\left(G_{1}, B_{1}\right)$ with $\operatorname{coz}(\hat{f}) \subset U$ and $\hat{T} \hat{f}(\xi) \neq 0$.

Let us remark that if $T$ is onto, then $\hat{G}_{20}=\hat{G}_{2}$.

Lemma 3.4. Given $\xi \in \hat{G}_{20}$, there exists a unique support point for $\xi$ in $\hat{G}_{1} \cup\{\infty\}$.

Proof. Suppose, contrary to what we claim, that there is no support point for a certain $\xi \in \hat{G}_{20}$, that is, for every $\gamma \in \hat{G}_{1} \cup\{\infty\}$, there exists a neighborhood $U$ such that if $f \in L^{1}\left(G_{1}, B_{1}\right)$ and $\operatorname{coz}(\hat{f}) \subset U$, then $\hat{T} \hat{f}(\xi)=0$. Since such neighborhoods form a cover of the compact $\hat{G}_{1} \cup\{\infty\}$, there exists a finite subcover, say $\left\{U_{1}, \ldots, U_{n}\right\}$. By [9, Lemma 1], we can find $\left\{f_{1}, \ldots, f_{n}\right\} \subset L^{1}\left(G_{1}\right)$ such that $\operatorname{coz}\left(\hat{f}_{i}\right) \subset U_{i}$ for $i=1, \ldots, n$ and $\sum_{i=1}^{n} \hat{f}_{i}=1$. Hence, given any $f \in L^{1}\left(G_{1}, B_{1}\right)$, we have $\hat{f}=\sum_{i=1}^{n} \hat{f} \hat{f}_{i}$ and $\hat{T}\left(\hat{f}_{i} \hat{f}\right)(\xi)=0$ for $i=1, \ldots, n$. Therefore, $\hat{T} \hat{f}(\xi)=0$ for all $f \in L^{1}\left(G_{1}, B_{1}\right)$, which contradicts the fact that $\xi \in \hat{G}_{20}$.

Let us suppose that $\gamma_{1}$ and $\gamma_{2}$ are two distinct support points for $\xi \in \hat{G}_{20}$. Let $V_{1}$ and $V_{2}$ be disjoint neighborhoods of $\gamma_{1}$ and $\gamma_{2}$, respectively. Then there exists $f_{1}, g_{1} \in L^{1}\left(G_{1}, B_{1}\right)$ such that $\operatorname{coz}\left(\hat{f}_{1}\right) \subset V_{1}$ and $\operatorname{coz}\left(\hat{f}_{2}\right) \subset V_{2}$, with $\hat{T} \hat{f}_{1}(\xi) \neq 0$ and $\hat{T} \hat{f}_{2}(\xi) \neq 0$, which contradicts the disjointness preserving property of $\hat{T}$.

Lemma 3.4 enables us to define a map $h: \hat{G}_{20} \longrightarrow \hat{G}_{1} \cup\{\infty\}$ which sends any $\xi \in \hat{G}_{20}$ to its support point.

Proposition 3.5. The map $h: \hat{G}_{20} \longrightarrow \hat{G}_{1} \cup\{\infty\}$ is continuous.

Proof. Let $\left(\xi_{d}\right)$ be a net in $\hat{G}_{20}$ converging to some $\xi_{0} \in \hat{G}_{20}$. Let $\left(h\left(\xi_{d^{\prime}}\right)\right)$ be a subnet of $\left(h\left(\xi_{d}\right)\right)$ which converges to some $\gamma_{0}$ in the compact space $\hat{G}_{1} \cup\{\infty\}$. Suppose, contrary to what we claim, that $h\left(\xi_{0}\right) \neq \gamma_{0}$. Let $U$ and $V$ be disjoint neighborhoods of $h\left(\xi_{0}\right)$ and $\gamma_{0}$, respectively. Then there exists $f \in L^{1}\left(G_{1}, B_{1}\right)$ such that $\hat{T} \hat{f}\left(\xi_{0}\right) \neq 0$ and $\operatorname{coz}(\hat{f}) \subset U$. On the other hand, as $\hat{T} \hat{f}$ is a continuous function, there must exist an index $d_{0}$ such $\hat{T} \hat{f}\left(\xi_{d_{0}}\right) \neq 0$ and $h\left(\xi_{d_{0}}\right) \in V$. Let $g \in L^{1}\left(G_{1}, B_{1}\right)$ such that $\hat{T} \hat{g}\left(\xi_{d_{0}}\right) \neq 0$ and $\operatorname{coz}(\hat{g}) \subset V$. Consequently, $\operatorname{coz}(\hat{f}) \cap \operatorname{coz}(\hat{g})=\emptyset$, but $\hat{T} \hat{f}\left(\xi_{d_{0}}\right) \neq 0$ and $\hat{T} \hat{g}\left(\xi_{d_{0}}\right) \neq 0$, which contradicts the disjointness preserving property of $\hat{T}$.

Proposition 3.6. Let $U$ be an open subset of $\hat{G}_{1} \cup\{\infty\}$ and let $f \in L^{1}\left(G_{1}, B_{1}\right)$. If $\left.\hat{f}\right|_{U} \equiv 0$, then $\left.\hat{T} \hat{f}\right|_{h^{-1}(U)} \equiv 0$.

Proof. Assume that $\hat{f}$ vanishes on an open subset $U$ of $\hat{G}_{1} \cup\{\infty\}$. If we take $\xi \in h^{-1}(U)$, then there exists $f^{\prime} \in L^{1}\left(G_{1}, B_{1}\right)$ with $\operatorname{coz}\left(\hat{f}^{\prime}\right) \subset U$ and $\hat{T} \hat{f}^{\prime}(\xi) \neq 0$. Since $\operatorname{coz}(\hat{f}) \cap \operatorname{coz}\left(\hat{f}^{\prime}\right)=\emptyset$, we infer that $\hat{T} \hat{f}(\xi)=0$. 
The following example, adapted from [10], shows that, in the vector-valued setting, unlike in the complex-valued case ([9]), the automatic continuity of $T$ cannot be obtained from its disjointness preserving property, even if $T$ is a biseparating bijection.

Example 3.7. Let $G$ be a trivial group consisting only of an identity element and let $c_{0}$ be the Banach algebra of all sequences which converge to zero. Then it is apparent that $A\left(\hat{G}, c_{0}\right)=C\left(\hat{G}, c_{0}\right)$. Let $e_{n}:=(0, \ldots, 0,1,0, \ldots) \in c_{0}$ and define a linear functional $\phi$ on $c_{0}$ such that $\phi\left(e_{n}\right)=n$, and a linear bijection $\Phi: c_{0} \longrightarrow c_{0}$ defined as $\Phi\left(\alpha_{1}, \alpha_{2}, \ldots\right):=\left(\alpha_{1}+\phi\left(\alpha_{1}, \alpha_{2}, \ldots\right), \alpha_{2}, \ldots\right)$. It is apparent that both are unbounded. Hence, if $\xi$ stands for the only element in $\hat{G}$, then we can define an unbounded biseparating bijection $\hat{T}: C\left(\hat{G}, c_{0}\right) \longrightarrow C\left(\hat{G}, c_{0}\right)$ as $\hat{T} \hat{f}(\xi)=\Phi[\hat{f}(\xi)]$ for all $\hat{f} \in C\left(\hat{G}, c_{0}\right)$.

Proposition 3.8. Assume that the disjointness preserving map $T$ is continuous and $\xi_{0} \in \hat{G}_{20}$. Then $h\left(\xi_{0}\right) \in \hat{G}_{1}$ and $\hat{T} \hat{f}\left(\xi_{0}\right)=0$ for all $f \in L^{1}\left(G_{1}, B_{1}\right)$ with $\hat{f}\left(h\left(\xi_{0}\right)\right)=0$.

Proof. Assume, contrary to what we claim, that $h\left(\xi_{0}\right)=\infty$. We first show that $\hat{T}\left(\hat{f}_{0} b\right)\left(\xi_{0}\right)=0$ for any $f_{0} \otimes b \in L^{1}\left(G_{1}\right) \otimes B_{1}$. By $[12,33.13]$, we know that $L^{1}\left(G_{1}\right)$ is Tauberian, that is, the set $\{f \in$ $L^{1}\left(G_{1}\right): \hat{f}$ has compact support $\}$ is a dense ideal in $L^{1}\left(G_{1}\right)$. Hence, given any $f_{0} \otimes b \in L^{1}\left(G_{1}, B_{1}\right)$, there exists a sequence of functions $\left(f_{n}\right) \subset L^{1}\left(G_{1}\right)$ whose Fourier transforms have compact support such that $\left(f_{n} \otimes b\right)$ converges to $f_{0} \otimes b$. For every $n \in \mathbb{N}$, we know that $\hat{f}_{n} b$ vanishes on a certain neighborhood of $\infty$. Hence, by Proposition 3.6, we have $\hat{T}\left(\hat{f}_{n} b\right)\left(\xi_{0}\right)=0$ for every $n \in \mathbb{N}$. Meantime, from the continuity of $T$ we get

$$
\left\|\hat{T}\left(\hat{f}_{n} b\right)-\hat{T}\left(\hat{f}_{0} b\right)\right\|_{\infty} \leq\left\|T\left(f_{n} \otimes b\right)-T\left(f_{0} \otimes b\right)\right\|_{1} \longrightarrow 0,
$$

which shows that $\hat{T}\left(\hat{f}_{0} b\right)\left(\xi_{0}\right)=\lim _{n \rightarrow \infty} \hat{T}\left(\hat{f}_{n} b\right)\left(\xi_{0}\right)=0$. Now for arbitrary $f \in L^{1}\left(G_{1}, B_{1}\right)$, as mentioned in Section 2, we have $f=\sum_{i=1}^{\infty} g_{i} \otimes b_{i}$, where $g_{i} \in L^{1}\left(G_{1}\right), b_{i} \in B_{1}$ and $\sum_{i=1}^{\infty}\left\|g_{i}\right\|_{1}\left\|b_{i}\right\|<\infty$. Thus, similarly, from the continuity of $T$ we conclude that $\hat{T} \hat{f}\left(\xi_{0}\right)=\sum_{i=1}^{\infty} \hat{T}\left(\hat{g}_{i} b_{i}\right)\left(\xi_{0}\right)=0$. Therefore we have $\hat{T} \hat{f}\left(\xi_{0}\right)=0$ for all $f \in L^{1}\left(G_{1}, B_{1}\right)$, which is a contradiction showing that $h\left(\xi_{0}\right) \neq \infty$.

Now we prove that $\hat{T} \hat{f}\left(\xi_{0}\right)=0$ for any $f \in L^{1}\left(G_{1}, B_{1}\right)$ with $\hat{f}\left(h\left(\xi_{0}\right)\right)=0$. As above, it is enough to consider the functions of the form $f_{1} \otimes b_{1}$ in the algebraic tensor product $L^{1}\left(G_{1}\right) \otimes B_{1}$. Assume, contrary to what we claim, that there exists $f_{0} \otimes b \in L^{1}\left(G_{1}, B_{1}\right)$ such that $\left(\hat{f}_{0} b\right)\left(h\left(\xi_{0}\right)\right)=0$ and $\hat{T}\left(\hat{f}_{0} b\right)\left(\xi_{0}\right) \neq 0$. From [20, Theorem 2.6.3], we can find, for each $n \in \mathbb{N}$, a function $k_{n}$ in $L^{1}\left(G_{1}\right)$ such that

(1) $\hat{k_{n}} \equiv 1$ on a neighborhood $V_{n}$ of $h\left(\xi_{0}\right)$,

(2) $\left\|\hat{k_{n}} \hat{f}_{0}\right\|_{\infty} \leq\left\|k_{n} * f_{0}\right\|_{1}<1 / n^{2}$.

Next we can define $g_{0}:=\sum_{n=1}^{\infty}\left(\left(k_{n} * f_{0}\right) \otimes b\right)$. Since $\left\|\left(k_{n} * f_{0}\right) \otimes b\right\|_{1}<\|b\| / n^{2}$ for every $n \in \mathbb{N}$, we infer that $g_{0}$ belongs to the Banach algebra $L^{1}\left(G_{1}, B_{1}\right)$. Furthermore, since $\hat{k_{n}} \hat{f}_{0} b \equiv \hat{f_{0}} b$ on 
$V_{n}$, which is a neighborhood of $h\left(\xi_{0}\right)$, by Proposition 3.6 and the additivity of $\hat{T}$, we deduce that $\hat{T}\left(\hat{k_{n}} \hat{f}_{0} b\right)\left(\xi_{0}\right)=\hat{T}\left(\hat{f}_{0} b\right)\left(\xi_{0}\right)$. As a consequence, from the continuity of $T$ it follows that

$$
\left\|\hat{T} \hat{g_{0}}\left(\xi_{0}\right)\right\|=\left\|\hat{T}\left(\sum_{n=1}^{\infty} \hat{k_{n}} \hat{f_{0}} b\right)\left(\xi_{0}\right)\right\|=\left\|\sum_{n=1}^{\infty} \hat{T}\left(\hat{f_{0}} b\right)\left(\xi_{0}\right)\right\|=\infty
$$

which is a contradiction.

Definition 3.9. Given $\xi \in \hat{G}_{20}$, let $w_{\xi}: B_{1} \longrightarrow B_{2}$ be defined as $w_{\xi}(b):=\hat{T}(\hat{e} b)(\xi)$ where $e \in L^{1}\left(G_{1}\right)$ and $\hat{e} \equiv 1$ on a certain neighborhood of $h(\xi)$. Furthermore, for any $\xi \in \hat{G}_{2} \backslash \hat{G}_{20}$, we define $w_{\xi} \equiv 0$.

We remark that by [20, Theorem 2.6.2], we can always find such function $e$ and by Proposition 3.6, we infer that the definition of $w_{\xi}$ does not depend on the choice of such $e$. So we can define the function $w$ by $w(\xi)=w_{\xi}$, and we will see in the next result that $w_{\xi}$ belongs to the space $\mathcal{L}\left(B_{1}, B_{2}\right)$ of continuous linear operators of $B_{1}$ into $B_{2}$ with the strong operator topology.

Meantime, we extend $h$ from $\hat{G}_{20}$ to $\hat{G}_{2}$, which we keep denoting by $h$, by assigning to $\xi$, for each $\xi \in \hat{G}_{2} \backslash \hat{G}_{20}$, an arbitrary point in $\hat{G}_{1}$.

Theorem 3.10. Let $T: L^{1}\left(G_{1}, B_{1}\right) \longrightarrow L^{1}\left(G_{2}, B_{2}\right)$ be a continuous disjointness preserving map. Then, there exist maps $h: \hat{G}_{2} \longrightarrow \hat{G}_{1}$ and $w: \hat{G}_{2} \longrightarrow \mathcal{L}\left(B_{1}, B_{2}\right)$ such that for any $\xi \in \hat{G}_{2}$ and any $f \in L^{1}\left(G_{1}, B_{1}\right)$, we have

$$
\hat{T} \hat{f}(\xi)=w_{\xi}[\hat{f}(h(\xi))]
$$

Moreover, $w$ and $h$ are continuous on $\hat{G}_{20}$.

Proof. Let $h$ and $w$ be defined as above. We claim that, for each $\xi \in \hat{G}_{20}$, the linear map $w_{\xi}$ is continuous, indeed, $w_{\xi} \in \mathcal{L}\left(B_{1}, B_{2}\right)$. To this end, let $e \in L^{1}(G)$ such that $\hat{e}(h(\xi))=1$ and $\|e\|_{1}<2$ (see [20, Theorem 2.6.3]). Then, if $b \in B_{1}$, we have $\left\|w_{\xi}(b)\right\|=\left\|w_{\xi}[(\hat{e} b)(h(\xi))]\right\|=\|\hat{T}(\hat{e} b)(\xi)\| \leq$ $\|\hat{T}(\hat{e} b)\|_{\infty} \leq\|T(e \otimes b)\|_{1} \leq\left\|T\left|\||| e \otimes b\|_{1} \leq 2\|T|\|||b| \mid\right.\right.$ thanks to the boundedness of $T$. Hence, $\left\|w_{\xi}\right\| \leq 2\|T\|$ for every $\xi \in \hat{G}_{20}$.

We now obtain the representation of $\hat{T}$. Assume first that $\xi \in \hat{G}_{20}$. Choose $e \in L^{1}(G)$ such that $\hat{e} \equiv 1$ on a certain neighborhood of $h(\xi)$ and let $f \in L^{1}\left(G_{1}\right)$. Since $((\hat{f}-\hat{f}(h(\xi)) \hat{e}) b)(h(\xi))=$ $(\hat{f} b-\hat{f}(h(\xi)) \hat{e} b)(h(\xi))=0$, we deduce, by Proposition 3.8, that $\hat{T}((\hat{f}-\hat{f}(h(\xi)) \hat{e}) b)(\xi)=0$. Hence $\hat{T}(\hat{f} b)(\xi)=\hat{T}(\hat{f}(h(\xi)) \hat{e} b)(\xi)=w_{\xi}[(\hat{f} b)(h(\xi))]$.

Given $f \in L^{1}\left(G_{1}, B_{1}\right)$, we know, as mentioned in Section 2, that $f=\sum_{i=1}^{\infty} f_{i} \otimes b_{i}$, where each $f_{i} \in L^{1}\left(G_{1}\right)$, each $b_{i} \in B_{1}$ and $\sum_{i=1}^{\infty}\left\|f_{i}\right\|_{1}\left\|b_{i}\right\|<\infty$. Hence, for any $\xi \in \hat{G}_{20}$, from the continuity of 
$T, w_{\xi}$ and the Fourier transform and also the above argument, we have

$$
\begin{aligned}
\hat{T} \hat{f}(\xi) & =\hat{T}\left(\sum_{i=1}^{\infty} \hat{f}_{i} b_{i}\right)(\xi)=\sum_{i=1}^{\infty} \hat{T}\left(\hat{f}_{i} b_{i}\right)(\xi) \\
& =\sum_{i=1}^{\infty} w_{\xi}\left[\left(\hat{f}_{i} b_{i}\right)(h(\xi))\right]=w_{\xi}\left[\sum_{i=1}^{\infty}\left(\hat{f}_{i} b_{i}\right)(h(\xi))\right] \\
& =w_{\xi}[\hat{f}(h(\xi))] .
\end{aligned}
$$

If we assume that $\xi \in \hat{G}_{2} \backslash \hat{G}_{20}$, then it is clear that $\hat{T} \hat{f}(\xi)=0=w_{\xi}[\hat{f}(h(\xi))]$.

Finally, to show the continuity of $w$, let $\left(\xi_{\alpha}\right)$ be a net in $\hat{G}_{20}$ converging to $\xi_{0} \in \hat{G}_{20}$. Choose $e \in L^{1}\left(G_{1}\right)$ such that $\hat{e} \equiv 1$ on a neighborhood $V$ of $h\left(\xi_{0}\right)$, by [20, Theorem 2.6.2]. Since $h$ is continuous on $\hat{G}_{20}$ by Proposition 3.5, we can assume, without loss of generality, that for each $\alpha$, $h\left(\xi_{\alpha}\right) \in V$. Hence, for each $b \in B_{1}$, from the continuity of $\hat{T}(\hat{e} b)$ it follows that

$$
w_{\xi_{\alpha}}(b)=\hat{T}(\hat{e} b)\left(\xi_{\alpha}\right) \longrightarrow \hat{T}(\hat{e} b)\left(\xi_{0}\right)=w_{\xi_{0}}(b)
$$

which shows that $w_{\xi_{\alpha}} \longrightarrow w_{\xi_{0}}$ is continuous strongly. Hence $w$ is continuous on $\hat{G}_{20}$.

It is worth mentioning that if $G_{2}$ is discrete, or equivalently, $\hat{G}_{2}$ is compact, then $w$ is continuous on $\hat{G}_{2}$. Indeed, in this case we have $w_{\xi}(b)=\hat{T}(b)(\xi)$ for all $\xi \in \hat{G}_{2}$ and $b \in B_{1}$, which easily yields the continuity of $w$ on $\hat{G}_{2}$.

As a corollary of the above theorem, we show that continuous disjointness preserving maps $T$ : $L^{1}\left(G_{1}, B_{1}\right) \longrightarrow B_{2}$ can be written as the composition of a linear map and an algebra homomorphism.

Corollary 3.11. Let $T: L^{1}\left(G_{1}, B_{1}\right) \longrightarrow B_{2}$ be a continuous disjointness preserving map. Then $T=W \circ H$, where $W: B_{1} \longrightarrow B_{2}$ is a linear map and $H: L^{1}\left(G_{1}, B_{1}\right) \longrightarrow B_{1}$ is an algebra homomorphism.

Proof. We assume, without loss of generality, that $T \neq 0$. We can identify $B_{2}$ with $L^{1}\left(\{g\}, B_{2}\right)$ for a certain singleton $\{g\}$. By Theorem 3.10, we know that $\hat{T} \hat{f}=w_{g}(\hat{f}(h(g)))$ for any $f \in L^{1}\left(G_{1}, B_{1}\right)$. Let us define a homomorphism $H: L^{1}\left(G_{1}, B_{1}\right) \longrightarrow B_{1}$ as follows: $\left.H(f)=\hat{f}(h(g))\right)$. It is clear that $\operatorname{ker}(H) \subseteq \operatorname{ker}(T)$.

Take an element $b_{1}$ in the range of $H$, that is, there exists $f_{1} \in L^{1}\left(G_{1}, B_{1}\right)$ such that $H\left(f_{1}\right)=b_{1}$. Define $w_{1}\left(b_{1}\right):=T\left(f_{1}\right)$, which is well defined and linear since $\operatorname{ker}(H) \subseteq \operatorname{ker}(T)$. Hence $w_{1}$ is a linear map defined from the range of $H$ into $B_{2}$ such that $T=w_{1} \circ H$. Such $w_{1}$ can be extended to a linear map $W$ which coincides with $w_{1}$ on the range of $H$, vanishes on its complement and $T=W \circ H$. 
Theorem 3.12. Let $T: L^{1}\left(G_{1}, B_{1}\right) \longrightarrow L^{1}\left(G_{2}, B_{2}\right)$ be a continuous biseparating map. Then, there exist a homeomorphism $h: \hat{G}_{2} \longrightarrow \hat{G}_{1}$ and a continuous function $w: \hat{G}_{2} \longrightarrow \mathcal{L}\left(B_{1}, B_{2}\right)$ such that for any $\xi \in \hat{G}_{2}$ and any $f \in L^{1}\left(G_{1}, B_{1}\right)$, we have

$$
\hat{T} \hat{f}(\xi)=w_{\xi}[\hat{f}(h(\xi))] .
$$

Moreover, for each $\xi \in \hat{G}_{2}, w_{\xi}$ is a bijective homeomorphism and especially, $B_{1}$ and $B_{2}$ are isomorphic as vector spaces.

Proof. Since $T$ is onto, then it is clear that $\hat{G}_{20}=\hat{G}_{2}$. Furthermore, by Theorem 3.10, there exist continuous maps $h: \hat{G}_{2} \longrightarrow \hat{G}_{1}$ and $w: \hat{G}_{2} \longrightarrow \mathcal{L}\left(B_{1}, B_{2}\right)$ such that $\hat{T} \hat{f}(\xi)=w_{\xi}[\hat{f}(h(\xi))]$ for all $\xi \in \hat{G}_{2}$ and $f \in L^{1}\left(G_{1}, B_{1}\right)$.

From the Open Mapping theorem we deduce that $T^{-1}$ is continuous. Then for the continuous disjointness preserving map $T^{-1}$, there exist two continuous maps $h^{\prime}: \hat{G}_{1} \longrightarrow \hat{G}_{2}$ and $w^{\prime}: \hat{G}_{1} \longrightarrow$ $\mathcal{L}\left(B_{2}, B_{1}\right)$ defined similarly as $h$ and $w$ for $T$ such that $\hat{T}^{-1} \hat{g}(\zeta)=w_{\zeta}^{\prime}\left[\hat{g}\left(h^{\prime}(\zeta)\right)\right]$ for all $\zeta \in \hat{G}_{1}$ and $g \in L^{1}\left(G_{2}, B_{2}\right)$, by Theorem 3.10 .

We claim that $h^{-1}=h^{\prime}$. To see this, we first show that for each $\xi \in \hat{G}_{2}, h^{\prime}(h(\xi))=\xi$. Suppose, on the contrary, that $\xi \in \hat{G}_{2}$ and $h^{\prime}(h(\xi)) \neq \xi$. Since $A\left(\hat{G}_{2}, B_{2}\right)$ separates the points of $\hat{G}_{2}$, there is $g \in L^{1}\left(G_{2}, B_{2}\right)$ such that $\hat{g}\left(h^{\prime}(h(\xi))\right)=0$ and $\hat{g}(\xi) \neq 0$. From the representation of $\hat{T}^{-1}$ it follows that $\hat{T}^{-1} \hat{g}(h(\xi))=0$. Then $\hat{g}(\xi)=0$ by the representation of $\hat{T}$, a contradiction which yields $h^{\prime}(h(\xi))=\xi$. Similarly, $h\left(h^{\prime}(\zeta)\right)=\zeta$ for all $\zeta \in \hat{G}_{1}$. Therefore, $h^{-1}=h^{\prime}$ which shows that $h$ is a homeomorphism.

We next prove that, for each $\xi \in \hat{G}_{2}, w_{\xi}$ is bijective. For this purpose, let $b \in B_{2} \backslash\{0\}$, and choose $g \in L^{1}\left(G_{2}, B_{2}\right)$ such that $\hat{g}(\xi)=b$. Then we have

$$
\begin{aligned}
b & =\hat{T}\left(\hat{T}^{-1} \hat{g}\right)(\xi)=w_{\xi}\left[\left(\hat{T}^{-1} \hat{g}\right)(h(\xi))\right] \\
& =w_{\xi}\left[w_{h(\xi)}^{\prime}(\hat{g}(\xi))\right]=w_{\xi}\left(w_{h(\xi)}^{\prime}(b)\right),
\end{aligned}
$$

and consequently, $b=w_{\xi}\left(w_{h(\xi)}^{\prime}(b)\right)$. Thus $w_{\xi} \circ w_{h(\xi)}^{\prime}$ is the identity operator on $B_{2}$. Similarly, one can see that $w_{h(\xi)}^{\prime} \circ w_{\xi}$ is the identity operator on $B_{1}$. Therefore, $w_{\xi}$ is a bijective map and so $B_{1}$ and $B_{2}$ are isomorphic as vector spaces. Moreover, by the Open Mapping theorem, $w_{\xi}^{-1}$ is continuous, i.e., $w_{\xi}$ is a homeomorphism.

\section{Compact disjointness PRESERVing MAPS on VECTOR-VAlued Group Algebras}

First let us state, adapted to our context, a vector-valued version of the Arzela-Ascoli theorem ([21, Theorem 2.1]):

Theorem 4.1. A subset $H$ of $C_{0}(\hat{G}, B)$ is relatively compact (or precompact) if and only if 
(1) $H$ is equicontinuous, that is, for every $\xi_{1} \in \hat{G}$ and every net $\left\{\xi_{\alpha}\right\}$ in $\hat{G}$ converging to $\xi_{1}$,

$$
\limsup _{\alpha}\left\{\left\|f\left(\xi_{\alpha}\right)-f\left(\xi_{1}\right)\right\|\right\}=0 .
$$

(2) $H(\xi):=\{f(\xi): f \in H\}$ is precompact in $B$ for every $\xi \in \hat{G}$.

(3) For every $\epsilon>0$, there exists a compact subset $K$ of $\hat{G}$ such that $\|f(\xi)\|<\epsilon$ for all $f \in H$ and all $\xi \in \hat{G} \backslash K$.

We will say that $T$ is compact if $\hat{T}$ transforms bounded sets into relatively compact ones. Let $w_{\xi}$, $\hat{G}_{20}, h$ and $w$ be defined as in the previous results. Then we can obtain the following characterization of compact continuous disjointness preserving maps:

Theorem 4.2. Let $T: L^{1}\left(G_{1}, B_{1}\right) \longrightarrow L^{1}\left(G_{2}, B_{2}\right)$ be a continuous disjointness preserving map. Then $T$ is compact if and only if

(1) $w_{\xi}$ is compact for every $\xi \in \hat{G}_{20}$.

(2) $h$ is locally constant on $\hat{G}_{20}$.

(3) For every $\epsilon>0$, there exists a compact subset $K$ of $\hat{G}_{2}$ such that $\left\|w_{\xi}(\hat{f}(h(\xi)))\right\|<\epsilon$ for all $f \in L^{1}\left(G_{1}, B_{1}\right)$ with $\|f\|_{1} \leq 1$ and all $\xi \in \hat{G}_{2} \backslash K$. Equivalently, the map $\xi \longrightarrow\left\|w_{\xi}\right\|$ vanishes at infinity.

(4) The map $w: \hat{G}_{2} \longrightarrow \mathcal{L}\left(B_{1}, B_{2}\right)$ is continuous when $\mathcal{L}\left(B_{1}, B_{2}\right)$ is equipped with the operator norm topology.

Proof. For the necessity, assume that $T$ is compact. Fix $\xi \in \hat{G}_{20}$ and let $\left\{b_{n}\right\}$ be a sequence in $\left\{b \in B_{1}:\|b\| \leq 1\right\}$. Let $f \in L^{1}\left(G_{1}\right)$ such that $\hat{f} \equiv 1$ on a certain neighborhood of $h(\xi)$ with $\|f\|_{1}<2\left(\left[20\right.\right.$, Theorem 2.6.3]), and define $f_{n}:=f \otimes b_{n}$. It is apparent that $\left\{\hat{f}_{n}\right\}$ is a bounded sequence. Since $T$ is compact, $\left\{\hat{T}\left(\hat{f}_{n}\right)\right\}$ is relatively compact. Hence by Theorems 3.10 and 4.1, we deduce that $\left\{\hat{T}\left(\hat{f}_{n}\right)(\xi)\right\}=\left\{w_{\xi}\left(\hat{f}_{n}(h(\xi))\right)\right\}=\left\{w_{\xi}\left(b_{n}\right)\right\}$ is relatively compact.

Assume, contrary to what we claim, that there exists $\xi_{0} \in \hat{G}_{20}$ such that $h$ is not constant on any open neighborhood $U$ of $\xi_{0}$. If we direct a neighborhood base at $\xi_{0}$ by inclusion, there exists a net $\left\{h\left(\xi_{U}\right)\right\}$ in $\hat{G}_{20}$ converging to $\xi_{0}$ and such that $h\left(\xi_{U}\right) \neq h\left(\xi_{0}\right)$ for all $U$. By [20, Theorem 2.6.3] we can find, for each $U, f_{U} \in L^{1}\left(G_{1}\right)$ with $\left\|f_{U}\right\|_{1}<2, \hat{f_{U}}\left(h\left(\xi_{0}\right)\right)=1$ and $\hat{f_{U}}\left(h\left(\xi_{U}\right)\right)=0$. Choose $b \in B_{1}$ such that $w_{\xi_{0}}(b) \neq 0$ and let $\mathcal{A}=\left\{f_{U} \otimes b\right\}_{U}$. It is clear that $\hat{\mathcal{A}}$ is bounded and, hence, $\hat{T}(\hat{\mathcal{A}})$ is relatively compact. Consequently, by Theorem 4.1, $\hat{T}(\hat{\mathcal{A}})$ is equicontinuous, but, for each $U$, we have

$$
\left\|\hat{T}\left(\hat{f_{U}} b\right)\left(\xi_{U}\right)-\hat{T}\left(\hat{f_{U}} b\right)\left(\xi_{0}\right)\right\|=\left\|w_{\xi_{0}}(b)\right\|>0
$$

a contradiction. 
Let $\mathcal{B}=\left\{f \in L^{1}\left(G_{1}, B_{1}\right):\|f\|_{1} \leq 1\right\}$. Since $\mathcal{B}$ is bounded and the Fourier transform is continuous, $\hat{\mathcal{B}}$ is also bounded. Now from the compactness of $\hat{T}$, we infer that $\hat{T}(\hat{\mathcal{B}})$ is relatively compact in $C_{0}\left(\hat{G}_{2}, B_{2}\right)$. By Theorem 4.1, we know that for every $\epsilon>0$, there exists a compact subset $K$ of $\hat{G}_{2}$ such that $\|\hat{T} \hat{f}(\xi)\|=\left\|w_{\xi}(\hat{f}(h(\xi)))\right\|<\epsilon$ for all $f \in \mathcal{B}$ and $\xi \in \hat{G}_{2} \backslash K$.

Now if $\xi \in \hat{G}_{2} \backslash K$ and $b \in B_{1}$ with $\|b\|=1$, then by considering $f=g \otimes b$, where $g$ is a function in $L^{1}\left(G_{1}\right)$ with $\|g\|_{1} \leq 2$ and $\hat{g}(h(\xi))=1$ ([20, Theorem 2.6.3]), from the previous paragraph it follows that

$$
\left\|w_{\xi}(b)\right\|=\left\|w_{\xi}(\hat{g}(h(\xi)) b)\right\|=\|\hat{T} \hat{g}(\xi)\|<2 \epsilon,
$$

and consequently, $\left\|w_{\xi}\right\| \leq 2 \epsilon$. Hence the map $\xi \longrightarrow\left\|w_{\xi}\right\|$ vanishes at infinity.

Next, we apply arguments similar to those in [15, Theorem 2] to show that condition (4) is valid. Contrary to what we claim, we assume that $w$ is not continuous at $\xi_{0} \in \hat{G}_{2}$. Hence there exists $\epsilon>0$ such that for each compact neighborhood $V$ of $\xi_{0}$ in $\hat{G}_{2}$, we can find $\xi_{V} \in V$ with $\left\|w_{\xi_{V}}-w_{\xi_{0}}\right\| \geq \epsilon$. Consequently, there is a net $\left\{e_{V}\right\}_{V}$ in $B_{1}$ with $\left\|e_{V}\right\|=1$ and $\left\|w_{\xi_{V}}\left(e_{V}\right)-w_{\xi_{0}}\left(e_{V}\right)\right\| \geq \epsilon$. Furthermore for each compact neighborhood $V$ of $\xi_{0}$ in $\hat{G}_{2}$, we can choose $g_{V} \in L^{1}\left(G_{1}, B_{1}\right)$ such that $\hat{g}_{V}\left(h\left(\xi_{V}\right)\right)=$ $\hat{g}_{V}\left(h\left(\xi_{0}\right)\right)=e_{V}$ and $\left\|g_{V}\right\|_{1} \leq 2([20$, Theorem 2.6.3]). Then from the representation of $\hat{T}$, it follows that

$$
\hat{T}\left(\hat{g}_{V}\right)\left(\xi_{V}\right)=w_{\xi_{V}}\left(e_{V}\right) \text { and } \hat{T}\left(\hat{g}_{V}\right)\left(\xi_{0}\right)=w_{\xi_{0}}\left(e_{V}\right) .
$$

Since $T$ is compact, there is a subnet of $\left\{\hat{g}_{V}\right\}_{V}$, which we keep denoting by $\left\{\hat{g}_{V}\right\}_{V}$, and a function $f$ in $L^{1}\left(G_{2}, B_{2}\right)$ such that $\left\|\hat{T}\left(\hat{g}_{V}\right)-\hat{f}\right\|_{\infty} \longrightarrow 0$. Hence we have

$$
\begin{aligned}
\left\|\hat{T}\left(\hat{g}_{V}\right)\left(\xi_{V}\right)-\hat{T}\left(\hat{g}_{V}\right)\left(\xi_{0}\right)\right\| & \leq\left\|\hat{T}\left(\hat{g}_{V}\right)\left(\xi_{V}\right)-\hat{f}\left(\xi_{V}\right)\right\|+\left\|\hat{f}\left(\xi_{V}\right)-\hat{f}\left(\xi_{0}\right)\right\|+\left\|\hat{f}\left(\xi_{0}\right)-\hat{T}\left(\hat{g}_{V}\right)\left(\xi_{0}\right)\right\| \\
& \leq\left\|\hat{T}\left(\hat{g}_{V}\right)-\hat{f}\right\|_{\infty}+\left\|\hat{f}\left(\xi_{V}\right)-\hat{f}\left(\xi_{0}\right)\right\|+\left\|\hat{f}\left(\xi_{0}\right)-\hat{T}\left(\hat{g}_{V}\right)\left(\xi_{0}\right)\right\| \longrightarrow 0,
\end{aligned}
$$

while

$$
\left\|\hat{T}\left(\hat{g}_{V}\right)\left(\xi_{V}\right)-\hat{T}\left(\hat{g}_{V}\right)\left(\xi_{0}\right)\right\|=\left\|w_{\xi_{V}}\left(e_{V}\right)-w_{\xi_{0}}\left(e_{V}\right)\right\| \geq \epsilon
$$

which is a contradiction. Therefore $w$ is continuous.

In order to prove the sufficiency, we must show that $\hat{T}(\hat{\mathcal{B}})$ is relatively compact in $C_{0}\left(\hat{G}_{2}, B_{2}\right)$ by checking the conditions (1)-(3) in Theorem 4.1. Fix $\xi_{0} \in \hat{G}_{20}$. Since the set $\left\{\hat{f}\left(h\left(\xi_{0}\right)\right): f \in \mathcal{B}\right\}$ is bounded in $B_{1}$ and $w_{\xi_{0}}$ is compact, we infer that $\left\{w_{\xi_{0}}\left(\hat{f}\left(h\left(\xi_{0}\right)\right)\right): f \in \mathcal{B}\right\}$ is relatively compact in $B_{2}$, which is to say that $\left\{\hat{T} \hat{f}\left(\xi_{0}\right): f \in \mathcal{B}\right\}$ is relatively compact in $B_{2}$.

Fix $\xi_{1} \in \hat{G}_{20}$. Let $\left\{\xi_{\alpha}\right\}$ be a net in $\hat{G}_{2}$ converging to $\xi_{1}$. Since $\hat{G}_{20}$ is an open subset of $\hat{G}_{2}$, then we can assume, without loss of generality, that for each $\alpha, \xi_{\alpha} \in \hat{G}_{20}$. Let $U$ be a neighborhood of $\xi_{1}$ where $h$ is constant. Hence, from a certain $\alpha_{0}, h\left(\xi_{\alpha}\right)=h\left(\xi_{1}\right)$ and, for all $f \in \mathcal{B}$, we have

$$
\left\|\hat{T} \hat{f}\left(\xi_{\alpha}\right)-\hat{T} \hat{f}\left(\xi_{1}\right)\right\|=\left\|w_{\xi_{\alpha}}\left(\hat{f}\left(h\left(\xi_{\alpha}\right)\right)\right)-w_{\xi_{1}}\left(\hat{f}\left(h\left(\xi_{1}\right)\right)\right)\right\| \leq\left\|w_{\xi_{\alpha}}-w_{\xi_{1}}\right\|
$$


for all $\alpha>\alpha_{0}$. It is, therefore, apparent, due to the continuity of $w$, that

$$
\limsup _{\alpha}\left\{\left\|\hat{\mathcal{B}} \hat{f}\left(\xi_{\alpha}\right)-\hat{T} \hat{f}\left(\xi_{1}\right)\right\|\right\}=0
$$

which yields the equicontinuity of $\hat{T}(\hat{\mathcal{B}})$ in $\xi_{1}$, which is arbitrary in $\hat{G}_{20}$.

Now assume that $\xi_{1} \in \hat{G}_{2} \backslash \hat{G}_{20}$. If $\left\{\xi_{\alpha}\right\}$ is a net in $\hat{G}_{2}$ converging to $\xi_{1}$, then for each $f \in \mathcal{B}$, we have

$$
\left\|\hat{T} \hat{f}\left(\xi_{\alpha}\right)-\hat{T} \hat{f}\left(\xi_{1}\right)\right\|=\left\|w_{\xi_{\alpha}}\left(\hat{f}\left(h\left(\xi_{\alpha}\right)\right)\right)\right\| \leq\left\|w_{\xi_{\alpha}}\right\|
$$

for all $\alpha$. Again from condition (4) it follows that $\lim _{\alpha} \sup _{f \in \mathcal{B}}\left\{\left\|\hat{T} \hat{f}\left(\xi_{\alpha}\right)\right\|\right\}=0$.

Finally, it is clear that condition (3) yields condition (3) in Theorem 4.1. As a consequence, $\hat{T}(\hat{\mathcal{B}})$ is relatively compact in $C_{0}\left(\hat{G}_{2}, B_{2}\right)$ and we are done.

Remark 4.3. It is known that when $\hat{G}$ is assumed to be compact, condition (3) in Theorem 4.1 is redundant (see e.g., [19, Theorem 47.1]). However, this is not the case in our (locally compact) context as the following example shows. Let us consider the following family of Fejer kernels in $L^{1}(\mathbb{R})$ :

$$
H=\left\{f_{n}(t)=n\left(\frac{\sin (n \pi t)}{n \pi t}\right)^{2}: n=1,2, \ldots\right\} .
$$

It is known (see e.g., [18, p.139]) that the family, $\hat{H}$, of Fourier transforms of the functions in $H$ turn out to be the following functions in $C_{0}(\mathbb{R})$ :

$$
\hat{f}_{n}(\xi)=1-\frac{|\xi|}{n}
$$

for $|\xi|<n$ and 0 otherwise. It can be easily checked both that $\hat{H}$ satisfies only conditions (1) and (2) in Theorem 4.1 and contains no convergent subsequence, which is to say that it cannot be relatively compact.

\section{REFERENCES}

[1] Y. Abramovich, Multiplicative representation of disjointness preserving operators. Indag. Math. 45 (1983), 265279 .

[2] Y. Abramovich, A.I. Veksler and A.V. Koldunov, On operators preserving disjointness. Soviet Math. Dokl. 248 (1983), 1033-1036.

[3] J. Araujo, Separating maps and linear isometries between some spaces of continuous functions. J. Math. Anal. Appl. 226 (1998), 23-39.

[4] W. Arendt, Spectral properties of Lamperti operators. Indiana Univ. Math. J. 32 (1983), 199-215.

[5] W. Arendt and D.R. Hart, The spectrum of quasi-invertible disjointness preserving operators. J. Funct. Anal. 68 (1986), 149-167.

[6] J.T. Chan, Operators with disjoint support property. J. Operator Theory 24 (2) (1990), 383-391.

[7] B. de Pagter, A note on disjointness preserving operators. Proc. Amer. Math. Soc. 90 (1984), 543-549. 
[8] J.J. Font and S. Hernández, Separating maps between locally compact spaces. Arch. Math. (Basel) 63 (1994), 158-165.

[9] J.J. Font and S. Hernández, Automatic continuity and representation of certain linear isomorphisms between group algebras. Indag. Math. 6 (4) (1995), 397-409.

[10] H-L. Gau, J-S. Jeang and N-C. Wong, Biseparating linear maps between continuous vector-valued function spaces. J. Aust. Math. Soc. 74 (2003), 101-109.

[11] A. Hausner, On a homomorphism between generalized group algebras. Bull. Amer. Math. Soc. 67 (1961), 138-141.

[12] E. Hewitt and K.A. Ross, Abstract Harmonic Analysis II. Springer Verlag, New York (1970).

[13] C. Huijsmans and B. de Pagter, Invertible disjointness preserving operators. Proc. Edinburgh Math. Soc. 37 (1993), 125-132.

[14] T.G. Honary, A. Nikou and A.H. Sanatpour, Disjointness preserving linear operators between Banach algebras of vector-valued functions. Banach J. Math. Anal. 8 (2) (2014), 93-106.

[15] J.E. Jamison and M. Rajagopalan, Weighted composition operator on C(X,E). J. Operator Theory 19 (2) (1988), 307-317.

[16] K. Jarosz, Automatic continuity of separating linear isomorphisms. Canad. Math. Bull. 33 (2) (1990), 139-144.

[17] K.B. Laursen and M. Neumann, Introduction to Local Spectral Theory. Oxford Univ. Press (2000).

[18] Y. Katznelson, An Introduction to Harmonic Analysis. Cambridge Univ. Press (2004).

[19] J.R. Munkres, Topology. M.I.T. Pearson (2000).

[20] W. Rudin, Fourier Analysis on Groups. Wiley-Interscience, New York (1962).

[21] W.M. Ruess and W.H. Summers, Compactness in spaces of vector-valued continuous functions and asymptotic almost periodicity. Math. Nachr. 135 (1988), 7-33.

[22] U.B. Tewari, M. Dutta and D.P. Vaidya, Multipliers of group algebras of vector-valued functions. Proc. Amer. Math. Soc. 81 (1981), 223-229.

Faculty of Mathematics, K. N. Toosi University of Technology, Tehran, 16315-1618, Iran

E-mail address: m.hosseini@kntu.ac.ir

Departamento de Matemáticas, Universitat Jaume i, Campus Riu Sec, 8029 AP, Castellón, Spain

E-mail address: font@mat.uji.es 